\title{
Características térmicas do solo observadas em cidades distintas do Estado da Paraíba
}

\author{
Soil thermal characteristics observed in different cities in the state of Paraíba
}

\author{
Júlio Mannuel Tavares Diniz ${ }^{1 *}$, Renata de Araújo Rafael ${ }^{1}$, José Fideles Filho², José Raimundo de Sousa Júnior ${ }^{3}$, \\ Almair de Albuquerque Fernandes ${ }^{4}$.
}

Resumo: A variação da temperatura do solo que uma planta consegue suportar é relativamente ampla, no entanto, seu desenvolvimento é seriamente comprometido a partir do momento em que solo passa a assumir temperaturas abaixo ou acima de certos valores limites. Até então, embora se saiba que a temperatura do solo é um dos fatores ambientais de maior importância para agricultura, poucos estudos acerca dessa variável foram realizados pela comunidade científica para os solos da região Nordeste do Brasil. Logo, o presente trabalho tem como objetivo o estudo das características térmicas do solo, a partir de dados coletados em duas cidades distintas do estado da Paraíba. Com base na análise dos resultados, verificou-se que o comportamento térmico do solo de ambas as cidades são bastante semelhantes, especialmente no que diz respeito às horas e aos meses de ocorrência das máximas e mínimas temperaturas. Á medida que se avança em profundidade constatou-se uma progressiva diminuição da variabilidade diária das temperaturas do solo. As estimativas da difusividade térmica do solo, obtidas mediante o emprego do método da amplitude e logarítmico, apresentaram satisfatória concordância.

Palavras-chave: Temperatura do solo, difusividade térmica, Paraíba, Nordeste do Brasil.

\begin{abstract}
The variation of soil temperature which a plant will tolerate is often quite broad, however, its development is seriously compromised from the moment in that the soil begins to assume temperatures below or above certain limits. Until then, although it is known that soil temperature is one of environmental factor of greatest importance to agriculture, few studies on this variable were performed by the scientific community for soils of Northeastern Brazil. Therefore, this work aims the study of soil thermal characteristics, from data collected in two different cities in the state of Paraíba. Based on the analysis of the results, it was found that the soil thermal behavior of both cities is quite similar, especially with respect to the hours and months of occurrence of maximum and minimum temperatures. It was found a progressive decrease of daily variability of soil temperatures as it advances in depth. The estimates of soil thermal diffusivity, obtained by employing the method of amplitude and logarithmic, showed satisfactory agreement.
\end{abstract}

Keywords: Soil temperature, thermal diffusivity, Paraíba, Northeastern Brazil.

\section{INTRODUÇÃO}

O solo é de fundamental importância para as plantas em virtude de suprir diariamente as suas necessidades de água e nutrientes, como também pelo fato de lhes propiciar ancoragem e estabilidade (OLIVEIRA et al., 2005). Para uma produção agrícola eficiente é importante compreender as características físicas do solo em que as plantas estão inseridas, a fim de reconhecer as limitações daquele ambiente e para melhorá-lo sempre que possível sem prejudicar a sua qualidade (GARDNER et al., 1999).

Em particular, a temperatura do solo é uma propriedade de natureza física que influi diretamente em uma série de processos ambientais relacionados às plantas tais como germinação de sementes, velocidade e duração de crescimento, desenvolvimento e atividade radicular, ocorrência e severidade de pragas, etc. (HILLEL, 2004). Segundo Mota (1983), a temperatura do solo possui maior importância ecológica para a vida vegetal do que a própria temperatura do ar.

$\mathrm{O}$ aquecimento demasiado do solo na fase inicial de estabelecimento das culturas compromete a absorção de nutrientes pelas plantas (CASTRO, 1989). As altas temperaturas também ocasionam efeitos nocivos sobre as raízes e a atividade microbiana (FURLANI et al., 2008). Johnson e Lowery (1985), ao realizarem estudo acerca dos efeitos de práticas de cultivo sobre as temperaturas e propriedades térmicas do solo, verificaram que a variação de $1^{\circ} \mathrm{C}$ na temperatura do solo pode afetar significativamente a taxa de crescimento do milho em regiões de clima temperado.

As magnitudes das temperaturas do solo estão em constantes alterações visto que este é continuamente perturbado pela "entrada" de calor mediante incidência de radiação solar como também pela "saída" através de processos físicos como a evaporação (LAL \& SHUKLA, 2004). Variações diurnas e sazonais na incidência de

\footnotetext{
*autor para correspondência

Recebido para publicação em 01/08/2013; aprovado em 30/09/2013

${ }^{1}$ Mestrando em Meteorologia, Unidade Acadêmica de Ciências Atmosféricas, Campina Grande - PB, Brasil. Email: julio_mannuel@hotmail.com

${ }^{2}$ Prof. Titular da Universidade Estadual da Paraíba. Departamento de Física, Campina Grande - PB, Brasil.

${ }^{3}$ Eng $^{\circ}$ Agrônomo, formado pela Universidade Federal de Campina Grande, Pombal - PB, Brasil.

${ }^{4}$ Licenciado em Geografia e Mestre em Sistemas Agroindustriais pela Universidade Federal de Campina Grande, Pombal - PB, Brasil.
} 
radiação solar fazem com que as condições térmicas do solo estejam em permanente alteração.

Medições de temperatura em diferentes profundidades do solo são freqüentemente realizadas em estações meteorológicas, no entanto, pouco se utiliza de tal acervo de dados uma vez que grande parte dos estudos não considera o fator térmico como limitante para a produção agrícola (atribuem maior ênfase ao fator hídrico). Informações acerca das temperaturas do solo, sua magnitude e forma de variação no tempo e espaço, são elementos de importância primordial para a determinação da taxa e direção dos processos físicos existentes no solo.

Quando uma mesma quantidade de energia está disponível para solos distintos o processo de aquecimento e resfriamento pode ser bastante variável em virtude de suas propriedades térmicas específicas. Basicamente, as propriedades térmicas dos solos que intervêm diretamente em seu regime de temperatura são denominadas de calor específico, condutividade térmica e difusividade térmica (WARRICK, 2001). Entre outros fatores, suas magnitudes dependem principalmente da composição do solo.

Em particular, a difusividade térmica do solo está diretamente relacionada à capacidade desse meio em conduzir calor através de seu perfil vertical, ou seja, é um indicativo da rapidez com que o calor difunde-se no solo. Como se verifica em Bellaver (2010), diferentes métodos vêm sendo propostos na busca de estimar a magnitude dessa propriedade. A difusividade térmica é função da constituição, granulometria, densidade e estrutura do solo (SILANS et al. 2006).

Sabendo-se da sua importância e uma vez que cada tipo de solo possui características próprias, faz-se necessário que sejam identificadas para cada situação particular as suas propriedades térmicas (DANELICHEN \& BIUDES, 2011). Até então, poucos estudos dessa natureza foram realizados pela comunidade científica para os solos da região Nordeste do Brasil. Logo, a presente pesquisa tem como objetivo específico, investigar a variabilidade espaço-temporal das temperaturas e difusividade térmica do solo de duas localidades distintas do estado da Paraíba.

\section{MATERIAL E MÉTODOS}

Para a elaboração da presente pesquisa foram utilizadas informações obtidas através de estações meteorológicas automáticas, localizadas em duas cidades distintas do estado da Paraíba, mais especificamente em Cajazeiras $\left(6,88^{\circ} \mathrm{S} ; 38,55^{\circ} \mathrm{W} ; 298 \mathrm{~m}\right)$ e João Pessoa $\left(7,11^{\circ} \mathrm{S} ; \quad 34,86^{\circ} \mathrm{W} ; \quad 7,4 \mathrm{~m}\right)$. Essas cidades foram selecionadas em virtude de suas características climáticas específicas e também devido à consistência e disponibilidade das informações. A localização geográfica de ambas as cidades pode ser observada na Figura-1.

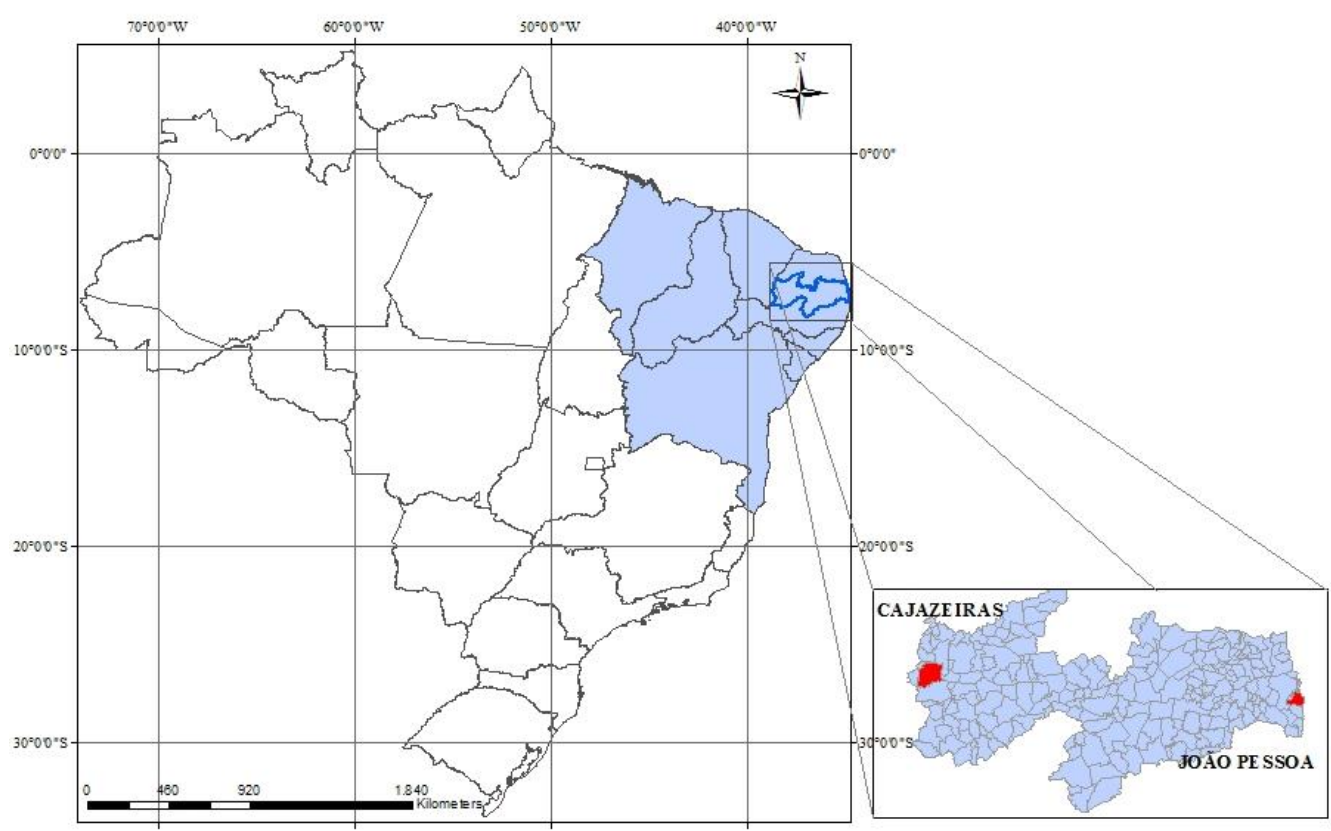

Figura 1. Localização das cidades de estudo.

Com o objetivo de monitorar o regime térmico diário do solo foram utilizados sensores de temperatura que operam durante 24 horas por dia, alojados em três profundidades distintas $(10,20$ e 50 centímetros). Pluviômetros, instalados de acordo com as especificações da Organização Mundial de Meteorologia (WMO), também foram utilizados para obter informação acerca da precipitação. Os dados empregados nesta pesquisa são provenientes dos meses de janeiro a junho do ano de 2013.

De acordo com Spiegel (1993), o grau ao qual os dados numéricos tendem a dispersar-se em torno de um valor médio chama-se variação ou dispersão dos dados. 
Dispõe-se de várias medidas de dispersão ou variação, sendo a utilizada nesta pesquisa denominada de coeficiente de variação (CV). A fim de verificar para cada profundidade a variabilidade diária das temperaturas do solo em torno dos respectivos valores médios (referentes a cada ciclo diário), fez-se uso da seguinte equação (THURMAN, 2012):

$$
\text { Coeficiente de Variação }=\frac{\mathrm{s}}{\overline{\mathrm{X}}} \times 100 \%
$$

Em que, s é o desvio padrão e $\overline{\mathrm{X}}$ são as médias diárias da temperatura do solo para cada profundidade. Segundo Larson e Farber (2004), pode-se interpretar o resultado do coeficiente de variação da seguinte maneira: quanto menor o CV mais homogêneo é o conjunto de dados e quanto maior o CV mais heterogêneo é o conjunto de dados.

A fim de estimar a difusividade térmica diária média mensal considerou-se o solo um meio homogêneo, isotrópico, sem fontes ou sumidouros de calor, restringindo a análise apenas aos processos que ocorrem na direção vertical. A partir dessas considerações irá ser aplicado o tratamento clássico de condução de calor a um meio homogêneo proposto por Fourier, que permite conhecer as amplitudes e fases da onda de calor (FIDELES FILHO, 1988).

Uma vez que a difusividade térmica do solo é calculada para certas camadas específicas do solo, faz-se necessário que sejam destacados os critérios adotados nesta pesquisa. Devido à disponibilidade de dados as estimativas foram realizadas para as porções do solo denominadas de camada 1 (estende-se desde 0,1 até 0,2 metros de profundidade), camada 2 (estende-se desde 0,2 até 0,5 metros de profundidade) e camada 3 (estende-se desde 0,1 até 0,5 metros de profundidade).

Mediante as informações e considerações anteriores empregam-se determinados métodos a fim de obter a magnitude da difusividade térmica do solo, cujos detalhes podem ser encontrados em Gao et al. (2009). Os métodos utilizados recebem a nomenclatura de método da amplitude, método do arco tangente e método logarítmico.

Para a estimativa da difusividade térmica do solo através do método da amplitude, utiliza-se a seguinte equação:

$$
K=\frac{\omega\left(Z_{2}-Z_{1}\right)^{2}}{2\left[\ln \left(A_{1} / A_{2}\right)\right]^{2}}
$$

$\mathrm{Na}$ equação acima $\omega$ é a velocidade angular da Terra $\left(7,27 \times 10^{-5} \mathrm{rad} / \mathrm{s}\right), \mathrm{A}_{1}$ e $\mathrm{A}_{2}$ são as amplitudes da onda de temperatura nas profundidades $\mathrm{Z}_{1}$ e $\mathrm{Z}_{2}$ (obtidas através das temperaturas observadas em cada profundidade), respectivamente. A estimativa a partir do método do arco tangente é obtida mediante o emprego da seguinte equação:

$$
\mathrm{K}=\frac{\omega\left(\mathrm{Z}_{2}-\mathrm{Z}_{1}\right)^{2}}{2\left\{\arctan \left[\frac{\left(\mathrm{T}_{1}-\mathrm{T}_{3}\right)\left(\mathrm{T}_{2}^{\circ}-\mathrm{T}_{4}^{\circ}\right)-\left(\mathrm{T}_{2}-\mathrm{T}_{4}\right)\left(\mathrm{T}_{1}^{\circ}-\mathrm{T}_{3}^{\circ}\right)}{\left(\mathrm{T}_{1}-\mathrm{T}_{3}\right)\left(\mathrm{T}_{1}-\mathrm{T}_{3}^{\circ}\right)+\left(\mathrm{T}_{2}-\mathrm{T}_{4}\right)\left(\mathrm{T}_{2}-\mathrm{T}_{4}\right)}\right]\right\}^{2}}
$$

Em que $\mathrm{T}_{1}, \mathrm{~T}_{2}, \mathrm{~T}_{3}$ e $\mathrm{T}_{4}$ são as temperaturas obtidas na profundidade $Z_{1}$ e $\mathrm{T}^{\prime}{ }_{1}, \mathrm{~T}_{2}, \mathrm{~T}_{3}{ }_{3}$ e $\mathrm{T}_{4}{ }_{4}$ são as temperaturas oriundas de $Z_{2}$. Em ambas as profundidades, deve-se utilizar as temperaturas observadas em intervalos de 6 em 6 horas (NERPIN \& CHUDNOVSKII, 1967).

O método logarítmico é análogo ao do arco tangente no que diz respeito ao espaçamento de 6 horas entre as temperaturas utilizadas para estimar a magnitude da difusividade térmica. Nesse método, a estimativa é realizada através da relação:

$$
\mathrm{K}=\left\{\frac{0,0121\left[\mathrm{Z}_{2}-\mathrm{Z}_{1}\right]}{\ln \left\{\left[\left(\mathrm{T}_{1}-\mathrm{T}_{3}\right)^{2}+\left(\mathrm{T}_{2}-\mathrm{T}_{4}\right)^{2}\right] /\left[\left(\mathrm{T}_{1}-\mathrm{T}_{3}^{\circ}\right)^{2}+\left(\mathrm{T}_{2}-\mathrm{T}_{4}\right)^{2}\right]\right.}\right\}^{2}
$$

Similarmente ao método anterior, $\mathrm{T}_{1}, \mathrm{~T}_{2}, \mathrm{~T}_{3}$ e $\mathrm{T}_{4}$ são as temperaturas obtidas na profundidade $Z_{1}$ e $T{ }_{1}, T^{\prime}{ }_{2}, T^{\prime}{ }_{3} \mathrm{e}$ $\mathrm{T}_{4}{ }_{4}$ são as temperaturas oriundas de $\mathrm{Z}_{2}$.

\section{RESULTADOS E DISCUSSÃO}

A partir dos dados de temperatura oriundos de três profundidades distintas do solo, obtidos especificamente durante os meses de janeiro a junho do ano 2013, tornouse possível o estudo das características térmicas do presente meio. A Figura-2 e Figura-3 expõem o comportamento térmico diário do solo observado nas cidades de João Pessoa e Cajazeiras, respectivamente. 

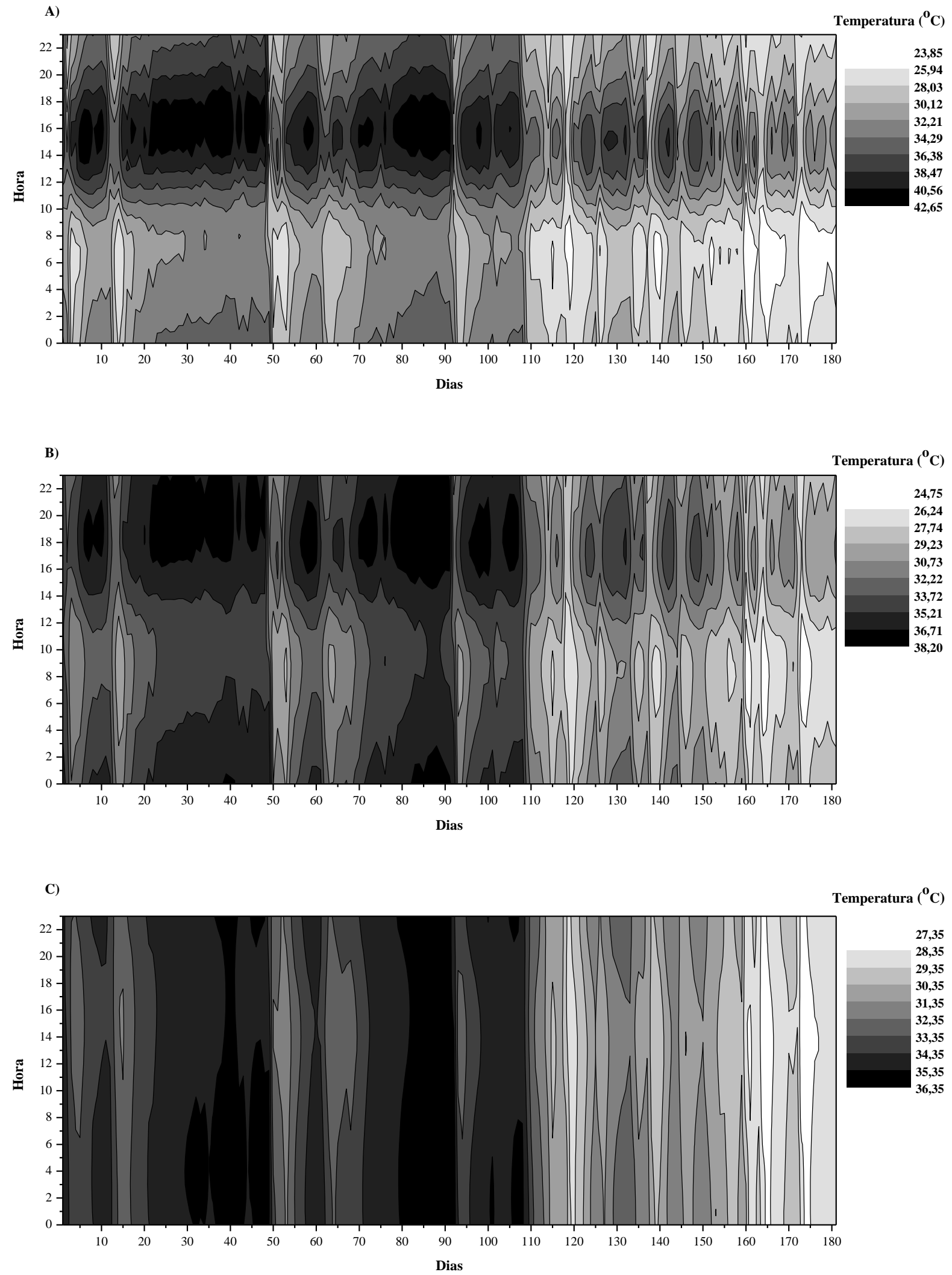

Figura 2. Comportamento térmico diário do solo de João Pessoa-PB, observado durante os meses de janeiro a junho do ano de 2013, nas seguintes profundidades: A) $10 \mathrm{~cm}$, B) $20 \mathrm{~cm} \mathrm{e} \mathrm{C)} 50 \mathrm{~cm}$. 

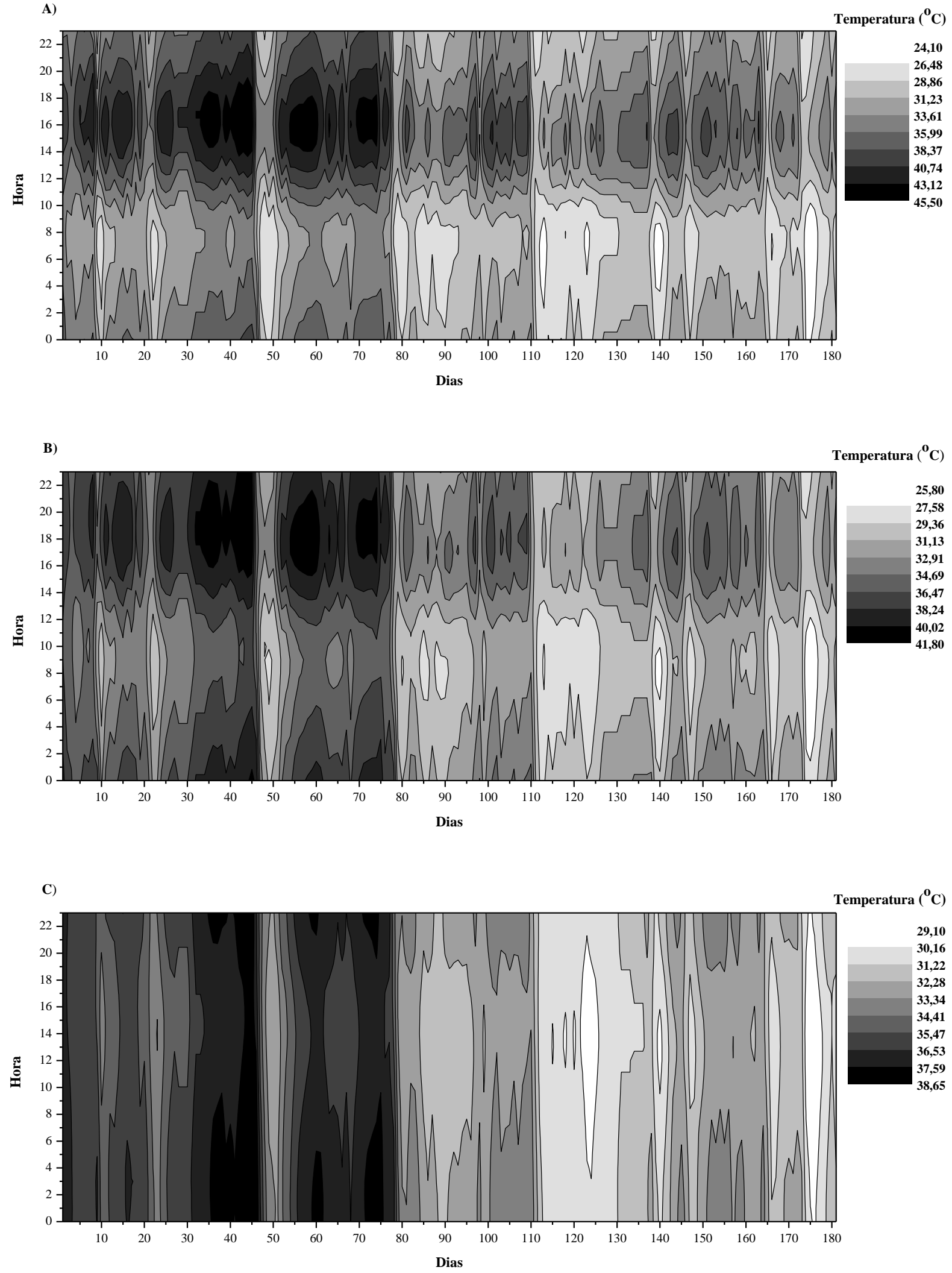

Figura 3. Comportamento térmico diário do solo de Cajazeiras-PB, observado durante os meses de janeiro a junho do ano de 2013, nas seguintes profundidades: A) $10 \mathrm{~cm}$, B) $20 \mathrm{~cm} \mathrm{e} \mathrm{C)} 50 \mathrm{~cm}$. 
Verifica-se, mediante a análise das figuras acima, que o comportamento térmico diário do solo de ambas as localidades são significativamente semelhantes. De modo geral, constata-se até meados das 10 horas da manhã o progressivo aumento das temperaturas à medida que se avança em profundidade, com os valores máximos sendo observados em $50 \mathrm{~cm}$. Após esse período, em conseqüência da incidência mais acentuada de radiação solar, a temperatura da superfície do solo e de regiões adjacentes passa a apresentar um significativo aumento de suas magnitudes com os valores máximos sendo observados em torno das 16 horas (Figura-2A e Figura3A). De acordo com Diniz et al. (2013a), o decréscimo das temperaturas observadas em seguida é conseqüência do decaimento da disponibilidade energética.

As temperaturas do solo apresentaram uma continua diminuição das suas magnitudes com o decorrer dos meses, sendo as mínimas registradas nas três profundidades durante o mês de Junho. Esse comportamento era esperado em virtude das observações terem sido iniciadas no verão (elevada disponibilidade energética) e finalizadas no inverno (baixa disponibilidade energética). Portanto, para este caso específico, pode-se deduzir que as temperaturas de maior magnitude são observadas de dezembro-março e as de menor entre junho-setembro. Uma vez que a disponibilidade energética pouco se altera ao longo do ano em regiões próximas ao equador geográfico (baixas latitudes), a variabilidade anual das condições térmicas do solo não é acentuada como a oriunda de demais regiões (médias e altas latitudes). Em ambas as localidades, a amplitude térmica para todo o período de análise não ultrapassa os $22^{\circ} \mathrm{C}$ (referente a região mais próxima a superfície).

Ao comparar à hora de ocorrência das máximas temperaturas do solo nas profundidades de $10 \mathrm{~cm}$ (Figura-
2A e Figura-3A) e $20 \mathrm{~cm}$ (Figura-2B e Figura-3B), verifica-se que esse fenômeno não ocorre simultaneamente em ambas as profundidades. Esse resultado indica que a onda de calor leva certo intervalo de tempo para se propagar no solo, variando de acordo com as suas propriedades específicas (constituição, capacidade calorífica, condutividade térmica, etc.). Gasparim et al. (2005), observaram comportamento semelhante ao realizar estudo acerca da temperatura no perfil do solo utilizando duas densidades de cobertura e solo nu. De acordo com Geiger (1980), esta mudança em comparação a região mais superficial é conseqüência do fluxo de calor no interior do solo acorrer de maneira relativamente lenta. A difusividade térmica, como mencionado anteriormente, fornece um indicativo da velocidade de avanço da onda de calor no solo. Mais adiante, serão apresentadas as estimativas da magnitude dessa grandeza física para ambas as localidades.

À medida que se avança em profundidade verifica-se que a amplitude térmica diária do solo tende a diminuir (variações bastante sutis), assumindo temperaturas quase que constantes em $50 \mathrm{~cm}$ (Figura-2C e Figura-3C). As regiões mais próximas a superfície apresentam maior amplitude térmica diária em decorrência da maior facilidade em ganhar (período diurno) e perder (período noturno) calor durante os ciclos diários (OLIVEIRA et al., 2010). Segundo Diniz et al. (2013b), uma maneira eficaz de verificar a variabilidade diária das temperaturas do solo com relação aos respectivos valores médios é através da estimativa do coeficiente de variação (CV). A Figura-4 apresenta o coeficiente de variação das temperaturas do solo para os ciclos diários e profundidades de estudo, juntamente com os respectivos totais pluviométricos (inseridos na busca de verificar a sua influência nos regimes de temperatura do solo). 

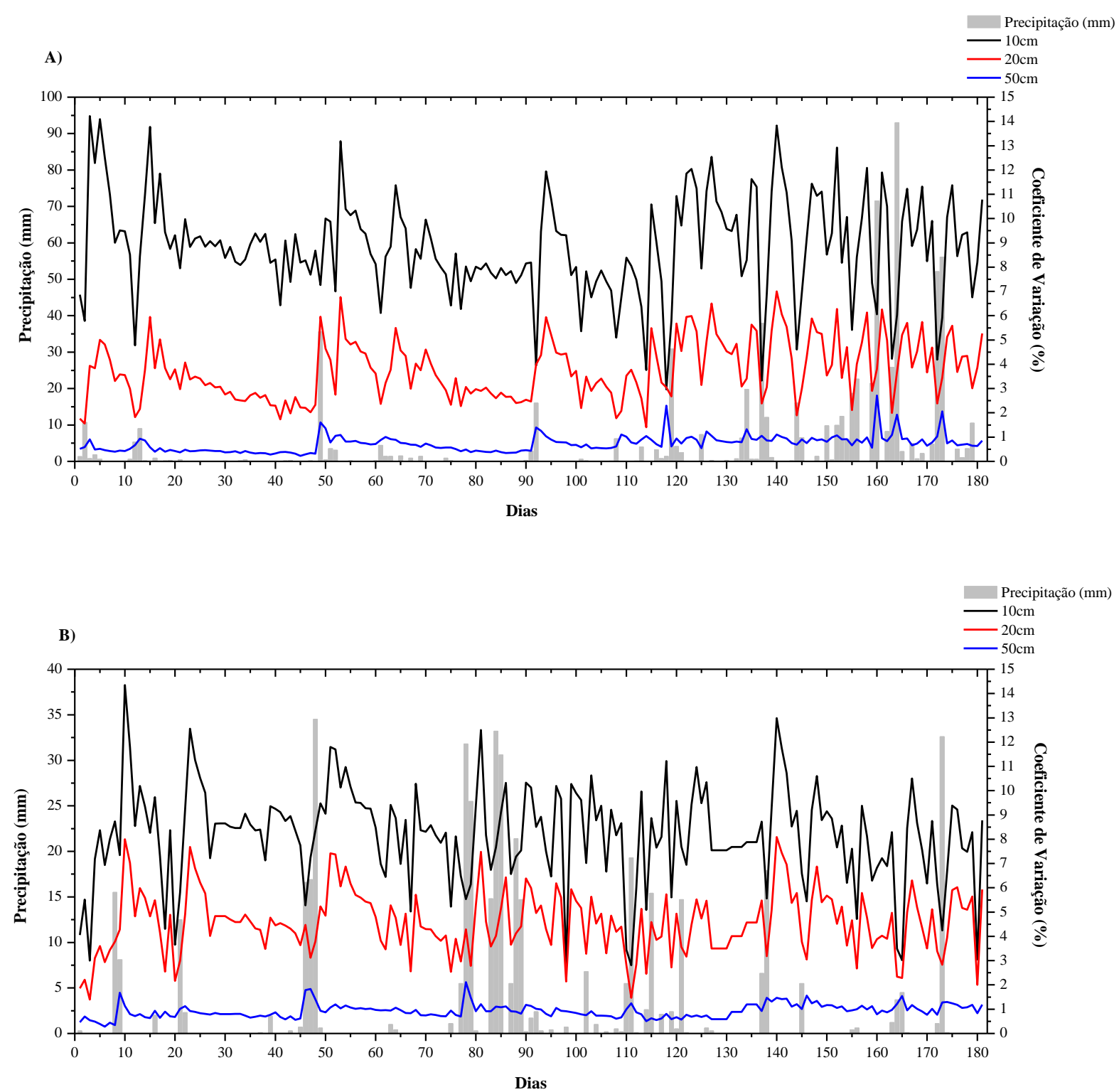

Figura 4. Coeficiente de variação das temperaturas do solo e totais pluviométricos, referentes aos ciclos diários compreendidos entre os meses de janeiro a junho do ano de 2013, obtidos para a cidade de João Pessoa (A) e Cajazeiras (B).

Constata-se com base na análise da figura acima que, conforme o observado anteriormente, a variabilidade diária das temperaturas do solo está inversamente relacionada à profundidade. As temperaturas se mantêm quase que inalteráveis ao longo dos ciclos diários na profundidade de $50 \mathrm{~cm}$, de modo que a magnitude do coeficiente de variação para o período de análise não ultrapassa os $3 \%$ (em geral, a amplitude térmica diária não excedeu os $3^{\circ} \mathrm{C}$ ). À medida que se aproxima da superfície observa-se que as condições térmicas do solo passam a se alterar de forma mais significativa durante os ciclos diários, de tal modo que o coeficiente de variação chega a assumir magnitudes em torno dos $7 \%$ na profundidade de
$20 \mathrm{~cm}$ (em geral, a amplitude térmica diária não excedeu os $8^{\circ} \mathrm{C}$ ). Ao comparar com as demais, as maiores magnitudes do coeficiente de variação são oriundas da profundidade de $10 \mathrm{~cm}$ (em torno dos $15 \%$ ), reflexo da sua elevada variabilidade térmica diária (verificada anteriormente na Figura-2A e Figura-3A).

Os fenômenos meteorológicos podem alterar de maneira significativa o comportamento térmico diário do solo. Em particular, com base na análise da Figura-4, verifica-se que a ocorrência de precipitação pluvial reduz consideravelmente a variabilidade diária das temperaturas do solo. A nebulosidade associada à precipitação impede que uma parcela significativa da radiação solar incida na 
superfície (devido aos processos de absorção e reflexão), de modo que as variações da temperatura do solo tendem a diminuir. A ocorrência de precipitação durante o período noturno não ocasionou efeitos significativos nos regimes de temperatura do solo de determinados ciclos diário, uma vez que estes apresentaram elevados totais pluviômetros e nenhuma alteração expressiva do coeficiente de variação.
Os dados de temperatura foram utilizados para estimar a difusividade térmica diária média mensal do respectivo meio. A Tabela-1 apresenta esses valores para três camadas específicas do solo, obtidos mediante o emprego de diferentes métodos (método da amplitude, método do arco tangente e método logarítmico).

Tabela 1. Difusividade térmica diária média mensal $\left(\mathrm{m}^{2} / \mathrm{s}\right)$, estimada para três camadas específicas do solo, a partir de métodos distintos.

\begin{tabular}{cccccccc} 
Mês & Método & & João Pessoa & & & Cajazeiras \\
& & Camada 1 & Camada 2 & Camada 3 & Camada 1 & Camada 2 & Camada 3 \\
\hline Jan. & Amplitude & $0,34 \mathrm{E}-06$ & $0,84 \mathrm{E}-06$ & $0,64 \mathrm{E}-06$ & $0,93 \mathrm{E}-06$ & $0,97 \mathrm{E}-06$ & $0,96 \mathrm{E}-07$ \\
& Arco tangente & $0,44 \mathrm{E}-06$ & $2,51 \mathrm{E}-06$ & $1,09 \mathrm{E}-04$ & $0,73 \mathrm{E}-06$ & $1,91 \mathrm{E}-06$ & $0,16 \mathrm{E}-04$ \\
& Logarítmico & $0,32 \mathrm{E}-06$ & $0,86 \mathrm{E}-06$ & $0,64 \mathrm{E}-06$ & $0,89 \mathrm{E}-06$ & $1,00 \mathrm{E}-06$ & $0,97 \mathrm{E}-06$ \\
\hline Fev. & Amplitude & $0,31 \mathrm{E}-06$ & $0,99 \mathrm{E}-06$ & $0,69 \mathrm{E}-06$ & $0,94 \mathrm{E}-06$ & $1,07 \mathrm{E}-06$ & $1,04 \mathrm{E}-06$ \\
& Arco tangente & $0,44 \mathrm{E}-06$ & $1,82 \mathrm{E}-06$ & $0,30 \mathrm{E}-04$ & $0,84 \mathrm{E}-06$ & $1,59 \mathrm{E}-06$ & $9,58 \mathrm{E}-06$ \\
& Logarítmico & $0,29 \mathrm{E}-06$ & $1,03 \mathrm{E}-06$ & $0,69 \mathrm{E}-06$ & $0,84 \mathrm{E}-06$ & $1,11 \mathrm{E}-06$ & $1,03 \mathrm{E}-06$ \\
\hline Mar. & Amplitude & $0,42 \mathrm{E}-06$ & $1,04 \mathrm{E}-06$ & $0,80 \mathrm{E}-06$ & $0,91 \mathrm{E}-06$ & $1,39 \mathrm{E}-06$ & $1,24 \mathrm{E}-06$ \\
& Arco tangente & $0,52 \mathrm{E}-06$ & $1,73 \mathrm{E}-06$ & $0,20 \mathrm{E}-04$ & $0,86 \mathrm{E}-06$ & $1,58 \mathrm{E}-06$ & $9,30 \mathrm{E}-06$ \\
& Logarítmico & $0,38 \mathrm{E}-06$ & $1,08 \mathrm{E}-06$ & $0,79 \mathrm{E}-06$ & $0,80 \mathrm{E}-06$ & $1,50 \mathrm{E}-06$ & $1,26 \mathrm{E}-06$ \\
\hline Abr. & Amplitude & $0,53 \mathrm{E}-06$ & $1,51 \mathrm{E}-06$ & $1,10 \mathrm{E}-06$ & $0,91 \mathrm{E}-06$ & $1,09 \mathrm{E}-06$ & $1,04 \mathrm{E}-06$ \\
& Arco tangente & $0,60 \mathrm{E}-06$ & $2,10 \mathrm{E}-06$ & $0,26 \mathrm{E}-04$ & $0,77 \mathrm{E}-06$ & $1,44 \mathrm{E}-06$ & $8,69 \mathrm{E}-06$ \\
& Logarítmico & $0,46 \mathrm{E}-06$ & $1,69 \mathrm{E}-06$ & $1,12 \mathrm{E}-06$ & $0,69 \mathrm{E}-06$ & $1,18 \mathrm{E}-06$ & $1,02 \mathrm{E}-06$ \\
\hline Mai. & Amplitude & $0,65 \mathrm{E}-06$ & $1,11 \mathrm{E}-06$ & $0,96 \mathrm{E}-06$ & $1,11 \mathrm{E}-06$ & $1,21 \mathrm{E}-06$ & $1,18 \mathrm{E}-06$ \\
& Arco tangente & $0,81 \mathrm{E}-06$ & $1,59 \mathrm{E}-06$ & $0,10 \mathrm{E}-04$ & $1,03 \mathrm{E}-06$ & $1,46 \mathrm{E}-06$ & $7,15 \mathrm{E}-06$ \\
& Logarítmico & $0,57 \mathrm{E}-06$ & $1,14 \mathrm{E}-06$ & $0,93 \mathrm{E}-06$ & $0,84 \mathrm{E}-06$ & $1,31 \mathrm{E}-06$ & $1,16 \mathrm{E}-06$ \\
\hline Jun. & Amplitude & $0,69 \mathrm{E}-06$ & $1,30 \mathrm{E}-06$ & $1,08 \mathrm{E}-06$ & $1,41 \mathrm{E}-06$ & $1,51 \mathrm{E}-06$ & $1,49 \mathrm{E}-06$ \\
& Arco tangente & $0,73 \mathrm{E}-06$ & $1,73 \mathrm{E}-06$ & $0,13 \mathrm{E}-04$ & $1,23 \mathrm{E}-06$ & $1,38 \mathrm{E}-06$ & $5,86 \mathrm{E}-06$ \\
& Logarítmico & $0,56 \mathrm{E}-06$ & $1,39 \mathrm{E}-06$ & $1,06 \mathrm{E}-06$ & $1,08 \mathrm{E}-06$ & $1,60 \mathrm{E}-06$ & $1,44 \mathrm{E}-06$ \\
\hline
\end{tabular}

A partir da análise da Tabela-1, verifica-se significativa semelhança entre os valores da difusividade térmica obtidos segundo os métodos da amplitude e logarítmico. Em particular, divergem das demais, as estimativas obtidas mediante o método do arco tangente para a camada 3. Rao et al. (2005), constataram fenômeno similar ao analisar o comportamento térmico do solo de Salvador-BA.

Devido à ordem de grandeza dos valores as "pequenas" divergências não comprometem os métodos, contudo, se faz necessário que os estudos a respeito dessa temática continuem sendo realizados na busca de desenvolver novos métodos e aprimorar os já existentes. De acordo com Silans et al. (1999), o método Harmônico proporciona bons resultados quando a condição de estabilidade da periodicidade é satisfeita, no entanto, durante dias com condições climáticas muito variáveis o mesmo deve ser substituído pelo método da Transformada de Laplace corrigida.

Os maiores valores da difusividade térmica do solo estão associadas à camada $2(0,2 \quad-\quad 0,5 \mathrm{~m} \quad \mathrm{de}$ profundidade). Logo, uma vez que a difusividade térmica está diretamente relacionada à condutividade térmica do meio, conclui-se que essa região do solo possui maior capacidade de conduzir calor através de seu perfil vertical. Essa característica pode está associada à existência de um maior teor de água nessa região específica, entretanto, esta afirmativa não pode ser tomada como verdade devido à falta de informações acerca da umidade do solo.

Em geral, ao contrário do que ocorre com as temperaturas, verifica-se que em ambas as localidades há um progressivo aumento das magnitudes da difusividade térmica do solo com o decorrer dos meses. Ao comparar à difusividade térmica de ambas as cidades, conclui-se que o solo de Cajazeiras possui maior capacidade de conduzir calor através de seu perfil vertical.

\section{CONCLUSÕES}

A partir da análise dos resultados pode-se concluir que o comportamento térmico do solo de ambas as cidades são bastante semelhantes, especialmente no que diz respeito às horas e meses de ocorrência das máximas e mínimas temperaturas. Em ambas as cidades, em decorrência da disponibilidade energética, as temperaturas de maior (menor) magnitude foram observadas no mês de Janeiro (Junho). Durante os ciclos diários, as máximas foram registradas em torno das 15 horas. Ainda com relação às magnitudes das temperaturas do solo, ao comparar ambas as cidades, verifica-se que as mais elevadas foram observadas em Cajazeiras.

À medida que se avança em profundidade verifica-se que as variações diárias das temperaturas do solo tendem a se tornar cada vez mais discretas, portanto, conclui-se que 
estas estão inversamente relacionadas. A ocorrência de precipitação afetou significativamente os regimes de temperatura, reduzindo de modo geral, a variabilidade térmica diária do solo. Portanto, os fenômenos meteorológicos não devem ser desconsiderados em estudos que se dedicam a quantificar e simular o regime de temperatura do solo.

Os métodos empregados para estimar a difusividade térmica do solo forneceram valores bastante semelhantes, com exceção daqueles obtidos pelo método do arco tangente para a camada 3 , indicando a consistência das metodologias. Em geral, os valores mais elevados da difusividade térmica do solo foram oriundos da camada 2. Novos estudos acerca dessa temática devem incluir informações acerca da umidade do solo, com o objetivo de verificar a sua influência nos regimes de temperatura.

\section{AGRADECIMENTOS}

Os autores agradecem a AESA (Agência Executiva de Gestão das Águas do Estado da Paraíba) pelo fornecimento dos dados utilizados neste estudo, bem como ao Conselho Nacional de Desenvolvimento Científico e Tecnológico (CNPq) e a Coordenação de Apoio ao Pessoal de Nível Superior (CAPES) pela concessão de bolsas de estudo.

\section{REFERÊNCIAS}

BELLAVER, V. Difusividade térmica do solo em área monodominante de cambará no norte do Pantanal Matogrossense. Dissertação (Mestrado em Física Ambiental) - UFMT, Mato Grosso. 54p. 2010.

CASTRO, O. M. Preparo do solo para a cultura do milho. Fundação Cargill. 41p. 1989.

DANELICHEN, V. H. M.; BIUDES, M. S. Avaliação da difusividade térmica de um solo no norte do Pantanal. Ciência e Natura, v. 33, n. 2, p. 227-240. 2011.

DINIZ, J. M. T.; SOUSA, E. P.; WANDERLEY, J. A. C.; FIDELES FILHO, J.; MARACAJÁ, P. B. Variabilidade diária da temperatura do solo: Um estudo de caso. Revista Verde de Agroecologia e Desenvolvimento Sustentável, v. 8, n. 1, p. 1-6. 2013a.

DINIZ, J. M. T; CARNEIRO, R. G.; ALVINO, F. C. G.; SOUSA, E. P.; SOUSA, E. P.; SOUSA JÚNIOR, J. R. Avaliação do comportamento térmico diário do solo de Campina Grande-PB. Agropecuária Científica no Semiárido, v. 9, n. 2, p. 77-82. 2013 b.

FIDELES FILHO, J. Estrutura térmica de solos do Nordeste do Brasil. Dissertação (Mestrado em Meteorologia) - UFPB, Campina Grande. 85p. 1988.
FURLANI, C. E. A.; GAMERO, C. A.; LEVIEN, R.; SILVA, R. P.; CORTEZ, J. W. Temperatura do solo em função do preparo do solo e do manejo da cobertura de inverno. Revista Brasileira de Ciência do Solo, v. 32, p. 375-380. 2008.

GAO, Z.; WANG, L.; HORTON, R. Comparison of six algorithms to determine the soil thermal diffusivity at a site in the Loess Plateau of China. Hydrology and Earth System Sciences Discussions, v. 6, p. 2247-2274. 2009.

GARDNER, C. M. K.; LARYEA, K. B.; UNGER, P. W. Soil physical constraints to plant growth and crop production. Land and Water Development Division. 96p. 1999.

GASPARIM, E.; RICIERI, R. P.; SILVA, S. L.; DALLACORT, R.; GNOATTO, E. Temperatura no perfil do solo utilizando duas densidades de cobertura e solo nu. Acta Scientiarum, v. 27, p. 107-115. 2005.

GEIGER, R. Manual de Micrometeorologia. Fundação Calouste Gulbenkian, 556p. 1980.

HILLEL, D. Introduction to environmental soil physics. Elsevier Science. 511p. 2004.

JOHNSON, M. D.; LOWERY, B. Effect of three conservation tillage practices on soil temperature and thermal properties. Soil Science Society of America Journal, v. 49, p. 1547-1552. 1985.

LAL, R.; SHUKLA, M. K. Principles of soil physics. Marcel Dekker. 699p. 2004.

LARSON, R.; FARBER, B. Estatística aplicada. Prentice Hall. 476 p. 2004.

MOTA, F. S. Meteorologia agrícola. Nobel S/A. 376p. 1983.

NERPIN, S. V.; CHUDNOVSKII, F. Physics of the soil. Keter Press. 466p. 1967.

OLIVEIRA, M. L.; RUIZ, H. A.; COSTA, L. M.; SCHAEFER, C. E. G. R. Flutuações de temperatura e umidade do solo em resposta à cobertura vegetal. Revista Brasileira de Engenharia Agrícola e Ambiental, v. 9, n. 4, p. 535-539. 2005.

OLIVEIRA, S. S.; FIDELES FILHO, J.; OLIVEIRA, S. V.; ARAÚJO, T. S. Difusividade térmica do solo de Campina Grande para dois períodos do ano. Revista de Geografia, v. 27, n. 2, p. 179-189. 2010.

RAO, T. V. R.; SILVA, B. B.; MOREIRA, A. A. Características térmicas do solo em Salvador, BA. Revista 
Brasileira de Engenharia Agrícola e Ambiental, v. 9, p. 554-559. 2005.

SILANS, A. M. B. P.; MONTENY, B. A.; LHOMME, J. P. Apparent soil thermal diffusivity, a case study: HAPEX - Sahel experiment. Agricultural and Forest Meteorology, v.81, p.201-216. 1999.

SILANS, A. P.; SILVA, F. M.; BARBOSA, F. A. R. Determinação in loco da difusividade térmica num solo da região de caatinga (PB). Revista Brasileira de Ciência do Solo, v. 30, p. 41-48. 2006.

SPIEGEL, M. R. Estatística. Makron Books. 643p. 1993.

THURMAN, P. W. Estatística. Saraiva. 232 p. 2012.

WARRICK, A. W. Soil physics companion. CRC Press. 403p. 2001. 